\title{
Sustainability of Nutraceuticals and Functional Foods
}

\section{Santad Wichienchot ${ }^{1}$ and Wan Rosli Wan Ishak ${ }^{2}$}

${ }^{1}$ Interdisciplinary Graduate School of Nutraceutical and Functional Food, Prince of Songkla University, Hat Yai, Songkhla, Thailand

${ }^{2}$ School of Health Sciences, Universiti Sains Malaysia Health Campus, Kubang Kerian, Kota Bharu, Kelantan, Malaysia

\begin{abstract}
SUMMARY
Sustainability challenges and issues in nutraceuticals and functional foods arena have influenced both consumers and the industrial sector. Though the present day can be termed as an era of nutraceuticals and functional foods, the knowledge on sustainability among the people involved is limited in the pharma-food industry. In this chapter, we provide a general overview of this subject, followed by discussion on the four pillars of sustainability and how these relate to the agricultural system, food safety and food security. Examples of nutraceuticals products, health benefits incurred, consumer trends and food choices as well as market values are all examined.
\end{abstract}

\subsection{Introduction}

Presently, many people know about using nutraceuticals and functional food products as health food and dietary supplement, but few know about the emerging issue of sustainability in nutraceuticals and functional foods. Nutraceuticals are well known as a food product that provides various significant health and medical benefits, including the prevention and treatment of certain diseases. It refers to the food or food ingredients that have provided specific physiological effects. Such products may range from specific diets to dietary supplements, herbal products, functional foods and functional drinks. A few examples of well-known commercially available nutraceutical products in the health food market include Betatene ${ }^{\bullet}$ (carotenoids), Xangold ${ }^{\odot}$ (lutein esters), Lipoec ${ }^{\odot}$ ( $\alpha$-lipoic acid), Gintex ${ }^{\odot}$ (Panax ginseng), Probio $^{\mathrm{TM}}$ (probiotic combination), Eyebil ${ }^{\odot}$ (bilberry) and Adovas ${ }^{\odot}$ (Adhatoda vasica). Dietary supplements refer to the preparations intended to compensate for the nutrients that otherwise may not be sufficiently present in the diet. Dietary supplements are categorized as food in some countries, while in other countries they are considered drugs or natural health products. Health foods are food considered beneficial to improve human health and as good source of normal healthy diet required for human nutrition (Ohama et al. 2006). Functional food is a food given an additional function (often one related to health promotion or 